\title{
AN INFINITE-DIMENSIONAL PRE-HILBERT SPACE NOT HOMEOMORPHIC TO ITS OWN SQUARE
}

\author{
ROMAN POL ${ }^{1}$
}

\begin{abstract}
Given an arbitrary infinite-dimensional separable complete linear metric space $X$, there exists a direct sum decomposition $X=V_{0} \oplus V_{1}$ such that each summand $V_{i}$ intersects every linearly independent Cantor set in $X$ (this decomposition can be considered as a linear analogue to the classical Bernstein's decomposition into totally imperfect sets).

THEOREM. Each summand $V$ of such a decomposition is not homeomorphic to its own square, and if $T: V \rightarrow V$ is a linear bounded operator, then either the kernel or the range of $T$ is finite-dimensional.

In the case of $X=I_{2}$ this provides an example of a space $V$ with the properties stated in the title, which answers a well-known question, cf. Arhangelskii [A, Problem 21] and Geoghegan [G, Problem (LS 12)].
\end{abstract}

1. Bernstein-like direct sum decompositions of infinite-dimensional complete linear separable metric spaces. Let $X$ be an infinite-dimensional separable complete linear metric space (cf. [B-P] for the terminology). In this note we shall consider direct sum decompositions

$$
X=V_{0} \oplus V_{1}
$$

such that

(2) if $C$ is a linearly independent Cantor set in $X$, then $V_{0} \cap C \neq \varnothing \neq V_{1} \cap C$;

here "Cantor set" means "topologically the Cantor set" and we shall call "Bernstein-like direct sum decomposition of $X$ " any decomposition of $X$ satisfying (1) and (2).

To obtain a Bernstein-like direct sum decomposition of $X$ one can follow a classical Bernstein's reasoning [ $\mathbf{K}_{\mathbf{1}}, \mathbf{I}, \S 36$, proof of Theorem 1]:

Let $\left\{C_{\alpha}: \alpha<2^{\omega}\right\}$ be all linearly independent Cantor sets in $X,{ }^{2}$ and let us choose inductively points $x_{\alpha}, y_{\alpha} \in C_{\alpha}$ such that at each stage $\beta$ of the construction the vectors $\left\{x_{\alpha}: \alpha<\beta\right\} \cup\left\{y_{\alpha}: \alpha<\beta\right\}$ are linearly independent; then let us add to the collection $\left\{x_{\alpha}: \alpha<2^{\omega}\right\} \cup\left\{y_{\alpha}: \alpha<2^{\omega}\right\}$ a set $A$ to obtain a linear basis in $X$; and finally, let $V_{0}$ be the linear span of the set $\left\{x_{\alpha}: \alpha<2^{\omega}\right\} \cup A$ and let $V_{1}$ be the linear span of the set $\left\{y_{\alpha}: \alpha<2^{\omega}\right\}$.

Received by the editors November 2, 1982.

1980 Mathematics Subject Classification. Primary 54F45.

Key words and phrases. Linear metric spaces, homeomorphisms, Bernstein's sets.

'This paper was written while the author was visiting the Free University, Amsterdam in September 1982. The author greatly acknowledges the hospitality of the Department of Mathematics of the University.

${ }^{2}$ If there were no such sets in $X$, (2) would turn simply into an empty condition; in fact, a theorem of Mycielski, see §2, shows that almost every Cantor set in $X$ is linearly independent. 


\section{The results.}

2.1. TheOREM. Let $X$ be an infinite-dimensional separable complete linear metric space and let $X=V_{0} \oplus V_{1}$ be a Bernstein-like direct sum decomposition of $X$ (see $\left.\S 1\right)$. Then each summand $V$ of the decomposition has the following properties:

(i) $V$ is of second category in $X$;

(ii) $V$ is not homeomorphic to its own square $V \times V$, and moreover,

(iii) if $V$ is homeomorphic to the product $E \times F$ of arbitrary spaces then either $E$ or $F$ contains a nonempty open finite-dimensional subspace;

(iv) if $T: V \rightarrow V$ is a linear bounded operator then either the kernel or the range of $T$ is finite-dimensional.

2.2. RemarK. If we take $X=l_{2}$, then the space $V$ described in Theorem 2.1 is a pre-Hilbert space not homeomorphic to its own square, which answers a well-known question, cf. Arhangelskiī [A, Problem 21] and Geoghegan [G, Problem (LS 12)]. Property (iv) shows also that in the pre-Hilbert space $V$ the only complementable linear subspaces are that of finite dimension or codimension.

3. A theorem of Mycielski on independent Cantor sets. The proof of Theorem 2.1 will be based on the following fact, being a fairly simple consequence of a general theorem on independent sets due to Mycielski [ $\left.\mathbf{M}_{\mathbf{1}}\right]$, cf., also $\left[\mathbf{M}_{\mathbf{2}}\right]$ and $\left[\mathbf{K}_{\mathbf{2}}\right]$.

3.1. Lemma. Let $A$ be an analytic set in a separable complete linear metric space $X$. If $A$ contains an uncountable linearly independent set $L$, then $A$ contains a linearly independent Cantor set.

Proof. Let $f: P \rightarrow A$ be a continuous map of the irrationals $P$ onto $A$, let $M$ be a subset of $P$ which is mapped by $f$ in a one-to-one way onto $L$ and let $Z$ be the set of all condensation points of $M$ in $P\left[\mathbf{K}_{1}, \S 18\right]$. Let $R_{n}=\left\{\left(x_{1}, \ldots, x_{n}\right) \in Z^{n}\right.$ : the vectors $f\left(x_{1}\right), \ldots, f\left(x_{n}\right)$ are linearly dependent $\}$. Then $Z$ is a completely metrizable space and each $R_{n}$ is closed in $Z^{n}$. Moreover, $M \cap Z$ is dense in $Z$ and if $x_{1}, \ldots, x_{n}$ are distinct points from $M$, then $\left(x_{1}, \ldots, x_{n}\right) \notin R_{n}$, so $R_{n}$ is nowhere dense in $Z^{n}$. Thus a theorem of Mycielski [ $\mathbf{M}_{\mathbf{1}}$, Theorem 1] and [ $\mathbf{M}_{\mathbf{2}}$, Theorem 1] guarantees an existence of a Cantor set $C \subset Z$ such that if $x_{1}, \ldots, x_{n}$ are distinct points from $C$ then $\left(x_{1}, \ldots, x_{n}\right) \notin R_{n}$, i.e. the vectors $f\left(x_{1}\right), \ldots, f\left(x_{n}\right)$ are linearly independent. It follows that $f$ maps $C$ homeomorphically onto a linearly independent Cantor set $f(C) \subset A$.

4. An auxiliary proposition. The following, rather special proposition, states a property of the space $V$ which is most essential in the proof of Theorem 2.1(iii) in $\S 5.2$ (cf. also Remark 7).

4.1. Proposition. Let $X=V_{0} \oplus V_{1}$ be a Bernstein-like direct sum decomposition as in Theorem 2.1 and let $V$ be any summand of this decomposition. Assume that $G$ is a $G_{\delta}$-set in $X$ containing $V$ and that $f: G \rightarrow Y$ is a continuous map onto a metrizable space $Y$. Then, either the image $f(V)$ is open modulo a first category set in $Y$, or there exists a point $y \in f(V)$ such that the fiber $f^{-1}(y)$ contains a relatively open nonempty finite-dimensional subset. 
Proof. At first, in (I) and (II), we shall recall some facts about the Effros Borel structure (the reader can consult Dellacherie [D, §2] or Christensen [Ch] for this topic); the essential part of the proof is the third one.

(I) Let $\mathbf{F}(G)$ be the set of all closed subsets of $G$ and let $\mathbf{B}(G)$ be the Effros Borel structure in $\mathbf{F}(G)$, i.e. the $\boldsymbol{\sigma}$-algebra generated by the sets

$$
\mathbf{U}=\{F \in \mathbf{F}(G): F \cap U \neq \varnothing\}, \quad U \text { being open in } G .
$$

Let $Z$ be a compact metrizable extension of $X$. Then the Effros Borel structure $\mathbf{B}(Z)$ in the hyperspace $\mathbf{F}(Z)$ of $Z$ coincides with the $\sigma$-algebra of Borel sets of the topological space $\mathbf{F}(Z)$ endowed with the exponential topology and the map $F \rightarrow \bar{F}$ from $\mathbf{F}(G)$ to $\mathbf{F}(Z)$ (the closure being taken in $Z$ ) is $(\mathbf{B}(G), \mathbf{B}(Z)$ )-measurable. Since the set of all finite-dimensional compact subsets of $Z$ is in $\mathbf{B}(Z)\left[\mathbf{K}_{\mathbf{1}}\right.$, Volume 2 , Theorem 4, p. 108], and since this is also true for the set of all compact subsets of $Z$ contained in $X$, the measurability of the map $F \rightarrow \bar{F}$ guarantees that

$\mathbf{E}=\{F \in \mathbf{F}(G)$ : the closure in $X$ of $F$ is compact and finite-dimensional $\} \in \mathbf{B}(G)$.

(II) Given an open set $W$ in $G$, let us define a map $\Phi: Y \rightarrow \mathbf{F}(G)$ by the formula

$$
\Phi(y)=\overline{f^{-1}(y) \cap W} .
$$

Let $\mathbf{A}(Y)$ be the $\sigma$-algebra in $Y$ generated by the family of analytic sets; recall, that by a classical theorem of Szpilrajn-Marczewski $\left[\mathbf{K}_{1}\right.$, II, $\S 35$, Corollary 1], each set in $\mathbf{A}(Y)$ is open modulo a first category set in $Y$. Let us verify that

$$
\Phi \text { is }(\mathbf{A}(Y), \mathbf{B}(G)) \text {-measurable. }
$$

Indeed, if $\mathbf{U}$ is a generator of the $\sigma$-algebra $\mathbf{B}(G)$ described in $(3)$, then $\Phi^{-1}(\mathbf{U})=$ $\left\{y \in Y: \overline{f^{-1}(y) \cap W} \cap U \neq \varnothing\right\}=f(U \cap W)$, which is an anlytic set.

(III) Let $W_{1}, W_{2}, \ldots$ be a countable base in $G$ and let, for each $i$,

$$
\Phi_{i}(y)=\overline{f^{-1}(y) \cap W_{i}} .
$$

It follows from (4) and (5) that

$$
E_{i}=\Phi_{i}^{-1}(\mathbf{E} \backslash\{\varnothing\}) \in \mathbf{A}(Y),
$$

so in particular, each $E_{i}$ is open modulo a first category set in $Y$. Let us check that

$$
Y \backslash f(V) \subset \bigcup_{i} E_{i} .
$$

If $y \in Y \backslash f(V)$, then $f^{-1}(y)$ is a $G_{\delta}$-set in $X$ disjoint from $V$ and hence, by Lemma 3.1 and property (2) of $V$, the linear space $L$ spanned by the set $f^{-1}(y)$ has the countable linear dimension. Thus $L$ is a union of countably many finite-dimensional linear subspaces, and therefore $f^{-1}(y)$ can be covered by countably many compact finite-dimensional sets $L_{1}, L_{2}, \ldots$ By the Baire category theorem, one of the sets $L_{k} \cap f^{-1}(y)$ has the nonempty interior in the space $f^{-1}(y)$, and therefore there exists an element $W_{i}$ in our base such that $\varnothing \neq f^{-1}(y) \cap W_{i} \subset L_{k}$. This means that (see (6) and (4)) $\Phi_{i}(y) \in \mathbf{E} \backslash\{\varnothing\}$, i.e. $y \in E_{i}$ (see (7)).

To end the proof it remains now to interpret properly the inclusion (8): either $f(V)=Y \backslash \cup_{i} E_{i}$ and then the set $f(V)$ is open modulo a first category set in $Y$, or 
else, there exists a point $y \in f(V) \cap E_{i}$, for some $i$, and then (see (4), (6) and (7)) $f^{-1}(y) \cap W_{i}$ is a nonempty open finite-dimensional set in the fiber $f^{-1}(y)$.

5. Proof of Theorem 2.1. Let $X=V_{0} \oplus V_{1}$ be a Bernstein-like direct sum decomposition as in Theorem 2.1 and let $V$ be one of the summands of this decomposition.

5.1. Proof of (i). Assume, toward a contradiction, that $V$ is of first category in $X$. Then there exists a dense $G_{\delta}$-set in $X$ disjoint from $V$ and, by virtue of Lemma 3.1 and property (2) of $V$, the linear space spanned by this $G_{\delta}$-set has the countable linear dimension. But then, the Baire category theorem easily yields an existence of a nonempty open finite-dimensional subset of $X$-a contradiction with infinite-dimensionality of $X$.

5.2. Proof of (iii). Assume that there exists a homeomorphism of the space $V$ onto the Cartesian product $E \times F$ of arbitrary spaces $E, F$; let $\tilde{E}$ and $\tilde{F}$ be completions of $E$ and $F$, respectively; and let us extend this homeomorphism, using the Lavrentieff theorem $\left[\mathbf{K}_{1}, \S 31, \mathrm{II}\right]$, to a homeomorphism $h: G \rightarrow H$ which maps a $G_{\delta}$-set $G$ in $X$ containing $V$ onto a $G_{\delta}$-set $H$ in the product $\tilde{E} \times \tilde{F}$ containing $E \times F$. Notice that $h(V)=E \times F$.

Let us assume that the space $F$ does not contain any nonempty open finite-dimensional sets. Let $p: \tilde{E} \times \tilde{F} \rightarrow \tilde{E}$ be the projection, let $Y=p(H)$ and let $f=p \circ h$ : $G \rightarrow Y$. Let us apply Proposition 4.1 to the map $f$.

The second possibility in the alternative asserted in Proposition 4.1 cannot occur, as for each $y \in f(V)=E$ we have $f^{-1}(y)=h^{-1}(\{y\} \times \tilde{F} \cap H)$, thus $F$ embeds topologically as a dense subspace in $f^{-1}(y)$ and hence there is no nonempty open finite-dimensional sets in the space $f^{-1}(y)$.

Therefore, the first part of this alternative must be true, i.e. $E$ is open modulo a first category set in $Y$. Now, $Y$ being an analytic set, $Y$ is open modulo a first category set in $\tilde{E}$ (the Szpilrajn-Marczewski theorem [ $\left.\mathbf{K}_{\mathbf{1}}, \mathrm{II}, \S 35\right]$ ) and hence $E$ is open modulo a set of first category in $\tilde{E}$. Moreover, $E$ is a space of second category, being a factor of the second category space $V$ (see $\$ 5.1$ ), and so $E$ contains a $G_{\delta^{\prime}}$-subset $A$ of $\tilde{E}$ of second category in $\tilde{E}$. Fix an $a \in F$ and put $A^{\prime}=h^{-1}(A \times\{a\})$, $E^{\prime}=h^{-1}(E \times\{a\})$. The set $A^{\prime}$ is a $G_{\delta}$-set in $X$ contained in $V$, hence by Lemma 3.1 and property (2) of $V$, the linear span of $A^{\prime}$ has the countable linear dimension, so $A^{\prime}$ is covered by countably many closed in $X$ finite-dimensional sets $L_{i}$. The restriction $f \mid E^{\prime}$ being a homeomorphism onto $E$, it follows that the closed finite-dimensional subsets $f\left(L_{i} \cap E^{\prime}\right)$ of $E$ cover the set $A$. Finally, the Baire category theorem applied to the completely metrizable space $A$ of second category in $E$ yields easily an existence of a nonempty open finite-dimensional subspace of $E$.

5.3. Proof of (iv). (We use here some improvements of the original reasoning, due to T. Dobrowolski.) Let $T: V \rightarrow V$ be a linear bounded operator and let $f$ be the extension of $T$ to a linear operator mapping the space $X$ into itself.

If there exists an $y \in f(X) \backslash V$, then the space $f^{-1}(y)=x+\operatorname{Ker} f$ ( $x$ being any vector with $f(x)=y$ ) is disjoint from $V$ and hence $\operatorname{Ker} f$ has the countable linear dimension, by Lemma 3.1 and property (2) of $V$, i.e. $\operatorname{Ker} f$ is finite-dimensional, being a complete space. Thus in this case $\operatorname{Ker} T \subset \operatorname{Ker} f$ is finite-dimensional.

The other alternative is that $f(X) \subset V$. Then $f(X)$ is an analytic linear subspace of $V$, and again by Lemma 3.1 and (2), $f(X)$ has the countable linear dimension. But 
the Baire category theorem applied to $X$ yields then easily that $f(X)$ is, in fact, finite-dimensional and so is the range of the operator $T$.

6. Remark. If one is only interested in a construction of a space $V$ with the properties stated in the title, then one can replace the main part of the proof given in $\S 5.2$ by the following simpler argument.

6.1. Lemma. Let $Z$ be a compact extension of the space $X$ and let $H$ be $a G_{\delta}$-set in $X \times Z$ containing the square $V \times V$ of the space $V$. Then the set $H^{*}$ of all $x \in X$ such that the vertical section $H(x)$ of $H$ at $x$ contains a topological copy of the Hilbert cube $I^{\omega}$ is an analytic set.

Proof. Let $(X \times Z) \backslash H=F_{1} \cup F_{2} \cup \cdots, F_{i}$ being a closed set in $X \times Z$, let $\mathbf{E}$ be the space of topological embeddings of $I^{\omega}$ into $Z$ endowed with the compact-open topology, and let $\mathbf{U}_{n}=\left\{(x, h) \in X \times \mathbf{E}: h\left(I^{\omega}\right) \cap F_{n}(x)=\varnothing\right\}, F_{n}(x)$ being the vertical section of $F_{n}$ at $x$. Then the sets $\mathbf{U}_{n}$ are open and $H^{*}$ is the projection of the $G_{\delta}$-set $\bigcap_{n} \mathbf{U}_{n}$ in $X \times \mathbf{E}$ onto the $X$-axis.

Now, let us adopt the notation of $\S 5.2$ with $E=F=V$ and $\tilde{E}=\tilde{F}=X$, where we assume also that $X$ is a Banach space.

If $x \notin V$, then $h^{-1}(\{x\} \times X \cap H)$ is a $G_{\delta}$-set in $X$ disjoint from $V$ and hence it does not contain a topological copy of $I^{\omega}$, by Lemma 3.1 and (2), and so the Hilbert cube does not embed into the vertical section $H(x)$. On the other hand, if $x \in V$, then $H(x)$ is a completely metrizable space containing an infinite-dimensional normed space $V$, and thus the Hilbert cube embeds into $H(x)$, cf. [B-P, Chapter VIII, Theorem 3.1].

It follows that $V$ coincides with the analytic set $H^{*}$ defined in Lemma 7.1. This however, contradicts Lemma 3.1 and property (2) of the space $V$.

6.2. RemarK. One can consider the above reasoning as a reminiscence of an old idea in the theory of analytic sets going back to Mazurkiewicz and Sierpiński, see Kuratowski [ $K_{1}$, VII, §43, Proof of Corollary 3].

AdDED IN Proof. W. Marciszewski recently constructed in a paper A pre-Hilbert space without any continuous map onto its own square, Bull. Polon. Acad. Sci. (to appear) a space with the properties indicated in the title.

\section{REFERENCES}

[A] A. V. Arhangelskiī, Structure and classification of topological spaces, Uspehi Mat. Nauk 33 (1978), 29-84.

[B-P] C. Bessaga and A. Pelczyński, Selected topics in infinite-dimensional topologl, PWN, Warsaw, 1975.

[Ch] J. P. R. Christensen, Topology and Borel structure, North-Holland, Amsterdam, 1974.

[D] C. Dellacherie, Un cours sur les ensembles analytiques, Analytic Sets, Academic Press, London, 1980.

[G] R. Geoghegan, Open problems in infinite-dimensional topologv, Topology Proc. 4 (1979).

$\left[\mathbf{K}_{1}\right]$ K. Kuratowski, Topology, Vols. I and II, PWN, Warsaw, 1966 and 1968.

$\left[\mathbf{K}_{2}\right] \ldots$. Applications of the Baire category method to the problem of independent sets, Fund. Math. 81 (1973), 65-72.

[ $\left.\mathbf{M}_{1}\right]$ J. Mycielski, Independent sets in topological algebras, Fund. Math. 55 (1964), 139-147.

$\left[\mathbf{M}_{2}\right] \ldots$, Almost every function is independent, Fund. Math. 81 (1973), 43-48.

Department of Mathematics, Warsaw University, PKin IXP., 00-901 Warszawa, Poland 